\title{
SYNTHESIS OF CLICKABLE COATING POLYMERS BY POST-POLYMERIZATION MODIFICATION: APPLICATION IN MICROARRAY TECHNOLOGY
}

\author{
Laura Sola ${ }^{1}$, Francesco Damin ${ }^{1}$, Paola Gagni ${ }^{1}$, Roberto Consonni ${ }^{2}$, Marcella Chiari ${ }^{1}$
}

\footnotetext{
${ }^{1}$ Istituto di Chimica del Riconoscimento Molecolare, CNR, Via Mario Bianco 9, 20131, Milano, Italy

${ }^{2}$ Istituto per lo Studio delle Macromolecole, CNR, Via Corti 12, 20133 Milano, Italy
} 


\section{Deprotection of thiolated oligonucleotides}

The thiolated oligonucleotides were diluted to a final concentration of $10 \mu \mathrm{M}$ into a $1 \mathrm{X}$ Tris-EDTA buffer containing $10 \mathrm{mM}$ of Tris(2-carboxyethyl)phosphine hydrochloride (TCEP). The solution was stirred continously for $1 \mathrm{~h}$ at $24^{\circ} \mathrm{C}$.

The deprotected oligonucleotides were precipitated by adding to the reaction mixture a solution containing sodium acetate and magnesium acetate tetrahydrate (final concentration $320 \mathrm{mM}$ and $9.8 \mathrm{mM}$ respectively) and ethanol (final concentration 70\%). Both the acetate solution and ethanol must be kept at $-20^{\circ} \mathrm{C}$. The oligonucleotides were allowed to precipitate for $2 \mathrm{~h}$ at $-80^{\circ} \mathrm{C}$, then the formed pellet was recovered by centrifugation (20 $\mathrm{min} 16000 \mathrm{rpm}$ ). Finally, the dried pellet was resuspended in DI water before use.
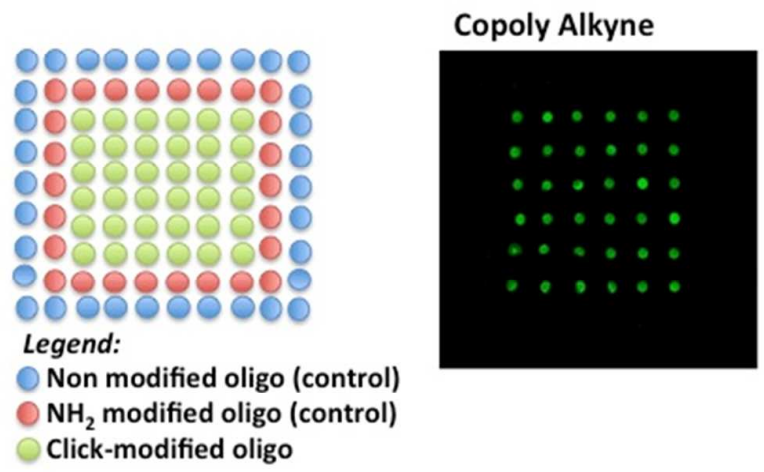

\section{Copoly Azide}
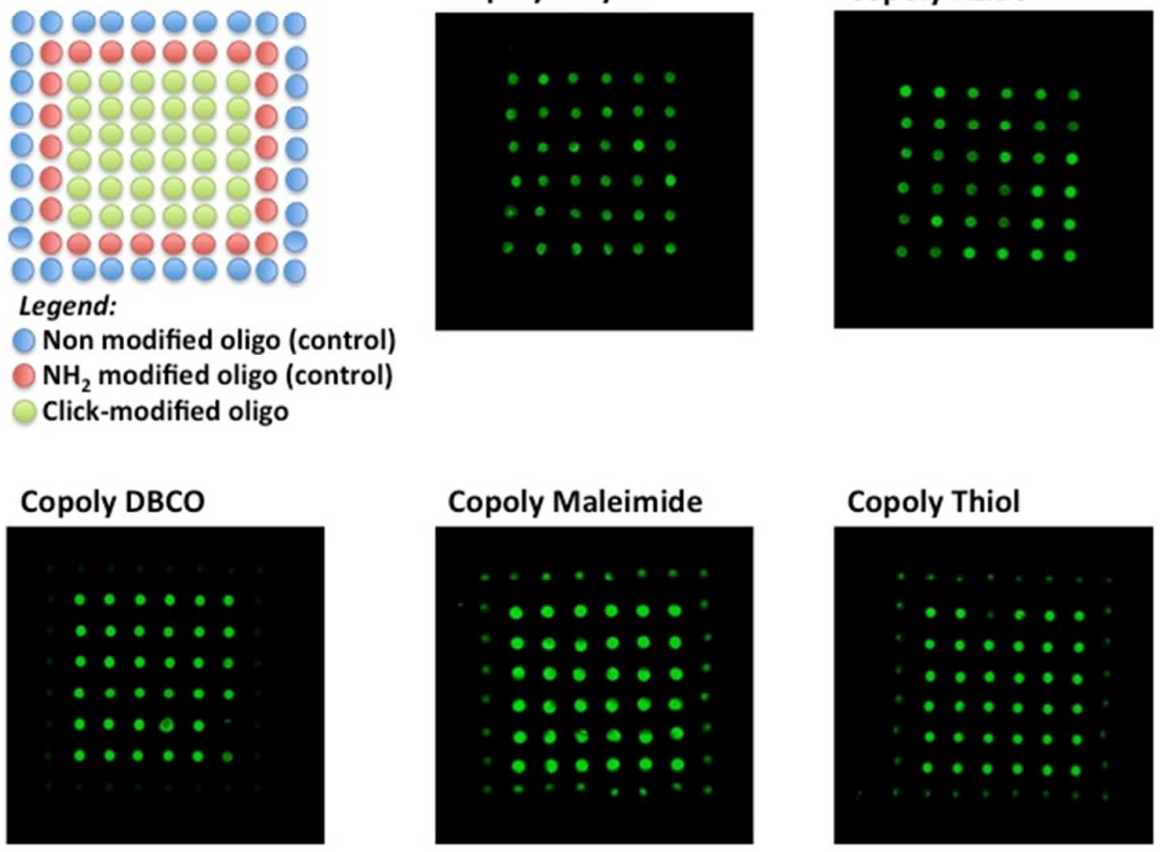

Figure S1 Spotting scheme and fluorescence images obtained after hybridization of the immobilized oligonucleotide with its complementary strand, labeled with Cy3. Each slide is coated 
with one of the new copolymer (as indicated in the Figure), obtained by postpolymerization modification of the parent copolymer Copoly (DMA-NAS-MAPS). 